\title{
Fabry disease: clinical and genotypic aspects of three cases in first degree relatives
}

Letícia Bueno Nunes da Silva ${ }^{1}$

Milvia Maria Simões e Silva Enokihara ${ }^{3}$

\author{
Thais Cardoso de Mello Tucunduva Badiz² \\ Adriana Maria Porro ${ }^{4}$
}

DOI: http://dx.doi.org/10.1590/abd1806-4841.20142785

\begin{abstract}
Fabry disease is an X-linked, lysosomal storage disease caused by the inherited deficiency of the enzyme $\alpha$-galactosidase A. The diagnosis is usually late, with renal, cardiovascular and/or cerebral complications that reduce life expectancy. Angiokeratomas are asymptomatic lesions present as the initial manifestation and usually less appreciated. Their detection is important for early diagnosis and institution of treatment with enzyme replacement therapy, which prevents late complications reducing morbidity and mortality. We report a case of a male teenager with acroparestesias and angiokeratomas. Family medical research discovered that his mother and brother had similar signs and symptoms and that the three patients had the same mutation in the gene encoding the enzyme, confirming the diagnosis.
\end{abstract}

Keywords: Angiokeratoma; Fabry disease; alpha-Galactosidase

\section{INTRODUCTION}

Fabry disease is a rare storage metabolic disease, linked to $\mathrm{X}$ chromosome, caused by deficiency of a- galactosidase A ( $a-G a l$ A) lysosomal hydrolase activity, leading to the accumulation of two neutral glycosphingolipids, globotriaosylceramide (GL-3 or Gb3) and digalactosylceramide, in several tissues, with progressive multisystemic damage..$^{1-5}$

The angiokeratomas are part of the initial clinical presentation, although asymptomatic. Angiokeratomas, acroparestesias and non-specific systemic symptoms are not initially associated with Fabry disease, which postpones the beginning of enzyme replacement therapy (ERT), increasing the risk of late complications. ${ }^{6}$

We report three cases of Fabry disease in two male brothers, in its classical form, as well as in the oligosymptomatic mother.

\section{CASES REPORT}

Case 1: 16-year-old male, with asymptomatic periumbilical lesions since his first years of life, progressing in number and size for the last 3 years. He presented right hypoacusia, tingling in hands and feet and burning plantar pain, which worsened with hot weather and physical activity.

At the dermatological exam he presented erythematous-violaceous papules of keratotic surface, grouped on the upper limbs, paravertebral, paraumbilical, inguinal, scrotum and penile regions, right thigh and knees (Figure 1). In the ophthalmological investigation the diagnosis was cornea verticillata (Figure 2). He presented hearing deficit in the right ear at audiometry. In complementary exams, he showed high 24hours proteinuria $(0.14$; VR $<0.05)$; remaining exams were normal (blood count, renal function, microalbuminuria, ECG, spirometry and cranial magnetic reso-

Received on 17.05.2013.

Approved by the Advisory Board and accepted for publication on 13.07.2013.

* Work performed at the Dermatology department, Escola Paulista de Medicina - Universidade Federal de São Paulo (EPM-UNIFESP) - São Paulo (SP), Brazil. Financial Support: none

Conflict of Interests: none

MD, Dermatologist - Graduate course in clinical dermatology, Dermatology Department, Escola Paulista de Medicina - Universidade Federal de São Paulo (EPM-UNIFESP) - São Paulo (SP), Brazil

MD, Dermatologist - Graduate course in Dermatoscopy, Dermatology Department, Escola Paulista de Medicina - Universidade Federal de São Paulo (EPMUNIFESP) - São Paulo (SP), Brazil.

MD, PhD - Dermatopathologist at the Dermatology and Pathology departments, Escola Paulista de Medicina - Universidade Federal de São Paulo (EPMUNIFESP) - São Paulo (SP), Brazil.

MD, PhD - Adjunct Professor of the Dermatology Department, Escola Paulista de Medicina - Universidade Federal de São Paulo (EPM-UNIFESP) - São Paulo (SP), Brazil. 
nance imaging-MRI). The deficiency of $\alpha$-Gal activity was confirmed in the plasma $(0.0 \mathrm{umol} / \mathrm{h}$ of protein; $>2.5)$ and leukocytes through paper filter $(0.16 \mathrm{nmol}$ / h / mg of protein; > 1.4). The biopy of umbilical lesion resulted compatible with angiokeratoma (Figure 3).
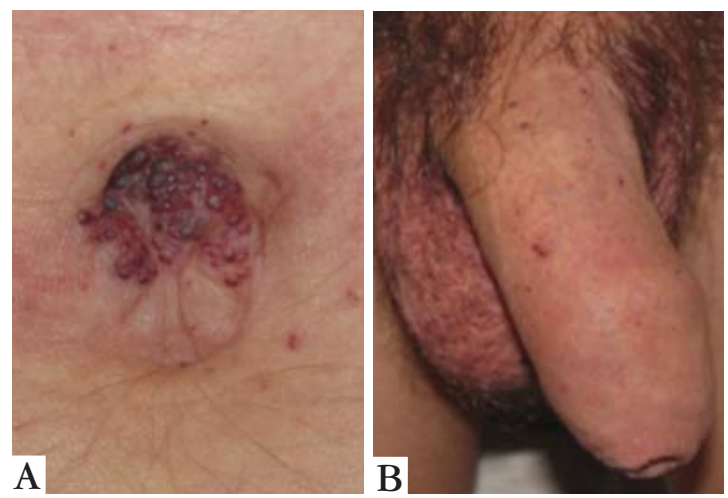

FIGURE 1: A. Keratotic erythematous-violaceous papules, distributed on the umbilical and paraumbilical areas. B. Keratotic violaceous papules distributed on scrotum and penile body

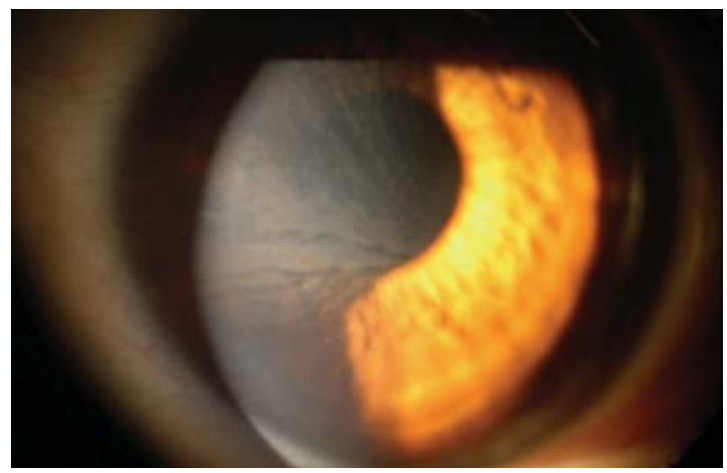

FIGURE 2: Cornea verticillata: Yellowish lines that converge to a spot close to central cornea region
Case 2: an 11-year-old boy presented burning pains in the lower limbs, which worsened with hot weather, although less intensively than in his brother's case (Case 1). During examination angiokeratomas were noticed, limited to the periumbilical region. He presented cornea verticillata, while the audiometry and other laboratory, electrocardiographic and imaging exams were normal. Enzyme $\alpha$ - Gal A deficiency was observed in the plasma and leukocytes (plasma: $0.11 \mathrm{umol} / \mathrm{h}$; leukocytes: $0.46 \mathrm{nmol} / \mathrm{mg} / \mathrm{h}$ ).

Case 3: a 39-year-old female, mother of patients 1 and 2, presented with mild acroparestesia and cornea verticillata, without skin lesions. Audiometry revealed diminished hearing in the right ear and slight loss in the left, discrete proteinuria (0.09) and microalbuminuria $(20.93 \mathrm{VR}<20)$. Normal blood count, renal function, ESR, ECG and cranial MRI. Dosage of $\alpha-G a l$ A activity in the plasma was within normal range (3.02 umol/h).

Genotyping of the 3 patients revealed a similar mutation: Exon 7 Arg $342 \mathrm{Gln}$. In the two-year followup, patient 1 presented worsening of lower limb pains and microangiopathy was detected in cranial MRI. Patient 2 started presenting proteinuria and increase of angiokeratomas in size, number and distribution. The mother remained stable. The three patients still have not started enzyme replacement due to family decision of postponing the beginning of treatment.

\section{DISCUSSION}

Fabry disease was originally described in 1898 as 'angiokeratoma corporis diffusum' by Anderson and Fabry. ${ }^{2,3}$ Later it was recognized as a systemic lysosomal storage disease, caused by a mutation of a-Gal gene $\mathrm{A}(\mathrm{Xq} 22.1)$, leading to alteration of enzyme expression. ${ }^{1,2}$ This deficiency leads to accumulation of neutral glycosphingolipids, particularly globotriaosylceramide (Gb3) and digalactosylceramide, mainly in the central nervous system, skin, heart, kidneys, auditory system and eyes. ${ }^{3,6}$

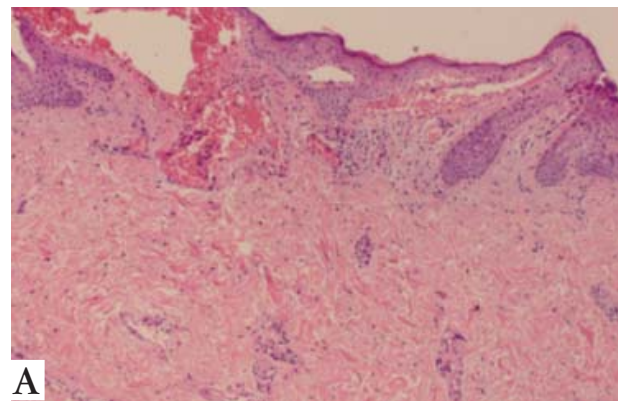

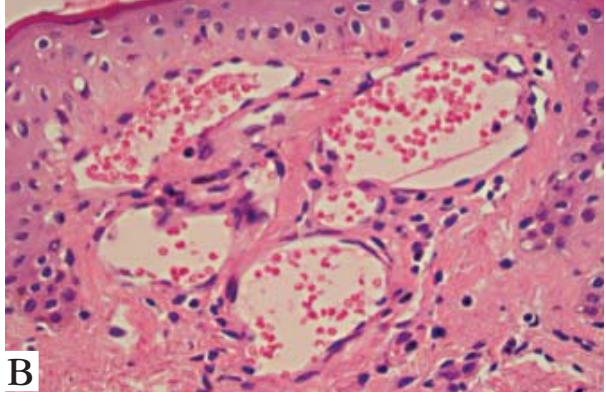

FIGURE 3

A. Proliferation of capillary vases, ectasia and congestion of the lumen in the papillary dermis, thickening of epidermis (HE100X).

B. Proliferation of capillary vases, ectasied, congested close to the dome of the papillary dermis (HE 400X) 
The clinical expression of the disease depends on the $\alpha$-galactosidase enzyme activity, whereas patients with residual enzymatic activity show a milder clinical picture, with predominance of cardiac alterations, possibly with minimal renal damage and absence of acroparestesia, which does not occur in the classical form of the disease, when the enzymatic activity is absent. ${ }^{2,7}$ This can be explained by more than 400 different gene mutations, some of them leading to some residual enzymatic activity varying between 2 to $25 \%{ }^{2}$

Even though it is considered a recessive disease linked to X chromosome, heterozygous women can manifest the disease symptoms in $70 \%$ of the cases, due to random activation of chromosome $X$, not being merely carriers of the mutation. ${ }^{2,3}$ This way, it is necessary to investigate and follow up female patients of families that carry Fabry disease, as was done with the patients' mother.

The classical form occurs in the male gender, as presented in the two brothers. The initial symptoms appeared in infancy, predominantly neurological, like acroparestesias, generalized pains, change of thermal sensitivity, hearing loss and hypohidrosis, but also gastrointestinal, including abdominal pain and change of intestinal habit. ${ }^{1,2,3,4}$ The cornea verticillata may be present since early childhood. ${ }^{6}$ Angiokeratomas are papules of red to purplish coloration, of varying sizes and tending to grow in number and size with age.
They are distributed mainly on the periumbilical and inguinal areas, in swimsuit form..$^{1,7}$ Angiokeratomas and cornea verticillata, when present, are a clue to an early diagnosis. ${ }^{7}$

The late manifestations occur in the third and fourth decades; the main ones are: chronic renal disease, cardiac arrhythmias and cerebral vascular accident, which contribute to reduce life expectancy of patients by 15 to 20 years. ${ }^{7}$ Early diagnosis is important for the establishment of enzymatic replacement treatment, diminishing Gb3 deposits in the vascular endothelium and plasma, with stabilization of renal function in children and postponing disease progress in adults. ${ }^{78,9}$ Recent studies suggest that, in spite of variable ERT response among patient subgroups, the treatment started before the occurrence of irreversible damage to organs could prevent late complications, with reduction of morbidity and mortality related to the disease. ${ }^{5,10}$

We reported three cases of Fabry disease in first-degree relatives. The index patient received the diagnosis at 16 years of age, despite the presence of angiokeratomas since early childhood, which demonstrates the importance of dermatologists and pediatricians knowing the condition to ensure that early enzymatic treatment is ministered, capable of avoiding late complications of the disease.

\section{REFERENCES}

1. Boggio P, Luna PC, Abad ME, Larralde M. Fabry Disease. An Bras Dermatol. 2009;84:367-76.

2. Schiffmann R. Fabry disease. Pharmacol Ther. 2009;122:65-77.

3. Martins AM, D'Almeida V, Kyosen So, Takata ET, Delgado AG, Gonçalves AM, et al. Guidelines to diagnosis and monitoring of Fabry Disease and review of treatment experiences. J Pediatr. 2009;155:S19-31.

4. Clarke JT. Narrative review: Fabry disease. Ann Intern Med. 2007;146:425-33.

5. Ramaswami U. Update on role of agalsidase alfa in management of Fabry disease. Drug Des Devel Ther. 2011;5:155-73.

6. Ramaswami U, Whybra C, Parini R, Pintos-Morell G, Mehta A, Sunder-Plassmann G, et al. Clinical manifestations of Fabry disease in children: Data from the Fabry Outcome Survey. Acta Paediatr. 2006;95:86-92.

7. Zarate YA, Hopkin JR. Fabry's disease. Lancet. 2008;372:1427-35.

8. Schiffmann R, Martin RA, Reimschisel T, Johnson K, Castaneda V, Lien YH, et al. Four-year prospective clinical trial of agalsidase alfa in children with Fabry disease. J Pediatr. 2010;156:832-7,837.e1.

9. Banikazemi M, Bultas J, Waldek S, Wilcox WR, Whitley CB, McDonald M, et al. Agalsidase-beta therapy for advanced Fabry disease: a randomized trial. Ann Intern Med. 2007;146:77-86

10. Alegra T, Vairo F, de Souza MV, Krug BC, Schwartz IV. Enzyme replacement therapy for Fabry disease: A systematic review and meta-analysis. Genet Mol Biol. 2012;35:947-54.

\author{
MAILING ADDRESS: \\ Letícia Bueno Nunes da Silva \\ Rua Borges Lagoa, 508 \\ Vila Clementino \\ 04038-001 - São Paulo - SP \\ Brazil \\ E-mail:leticiabns@yahoo.com.br
}

How to cite this article: Silva LBN, Tucunduva Badiz TCM, Enokihara MMSS, Porro AM. Fabry disease: clinical and genotypic aspects of three cases in first degree relatives. An Bras Dermatol. 2014;89(1):141-3. 